Volume 3

Issue 4 -- Cardiovascular Aging

$11-11-2016$

\title{
Prevalence of Prescription Opioid Abuse in Patients With Pain
}

Samuel Godana

Ankoor Biswas

Nebiyu Biru

Abraham Getenet

Biana Leybishkis

Follow this and additional works at: https://aah.org/jpcrr

Part of the Internal Medicine Commons, Pharmacy Administration, Policy and Regulation Commons, and the Substance Abuse and Addiction Commons

\section{Recommended Citation}

Godana S, Biswas A, Biru N, Getenet A, Leybishkis B. Prevalence of prescription opioid abuse in patients with pain. J Patient Cent Res Rev. 2016;3:239.

Published quarterly by Midwest-based health system Advocate Aurora Health and indexed in PubMed Central, the Journal of Patient-Centered Research and Reviews (JPCRR) is an open access, peer-reviewed medical journal focused on disseminating scholarly works devoted to improving patient-centered care practices, health outcomes, and the patient experience. 
2: Instructional procedures manual was created for 15 common procedures and provided to clinics. Phase 3: Postintervention survey was distributed after providers had access to procedures manual.

Results: Presurvey data revealed the majority of respondents felt neutral about their ability to perform procedures. However, residents felt more comfortable performing procedures than faculty. Postsurvey data revealed that a minority of respondents were aware of or used the procedures manual.

Conclusion: Our study demonstrated that a targeted intervention to improve the efficiency of procedure performance in the clinic setting was not well utilized. Therefore, a definitive conclusion about the ability of the intervention to improve the efficiency of procedure performance was not able to be made. Future areas of focus include better promotion of and ease of access to any selected intervention to improve efficiency of procedure performance.

\section{Prevalence of Prescription Opioid Abuse in Patients With Pain}

Samuel Godana, Ankoor Biswas, Nebiyu Biru, Abraham Getenet, Biana Leybishkis

\section{Department of Internal Medicine, Aurora Sinai Medical Center; Department of Internal Medicine, Aurora UW Medical Group}

Background: Studies showed that $20 \%$ to $30 \%$ of opioid analgesic drugs prescribed for chronic pain in the United States are misused, while the rate of opioid addiction is approximately $10 \%$. The study describes methods to identify high-risk behavior and forward recommendations to decrease opioid abuse.

Purpose: To assess prevalence and correlate multiple variables with opioid abuse and to forward appropriate recommendation to decrease the prevalence of opioid abuse in the primary care setting.

Methods: A prospective study was conducted at a primary health care clinic, Aurora Sinai Medical Center (Milwaukee, WI). Over 6 months, a total of 49 consecutive patients who take opioids were included in the study. Screener and Opioid Assessment for Patients with Pain-Revised (SOAPP-R), with a cutoff score of 18 (out of 24), is used to screen abuse/aberrant drug use. Additional variables were obtained by patient chart review. Data analysis was done using Minitab analysis software. Results: A total of 49 patients (23 males, 26 females) were included in the study. Mean age of patients was $45.7 \pm 7$ years; 22 (44.9\%) patients were on hydrocodone, $16(32.7 \%)$ on oxycodone, $7(14.3 \%)$ on tramadol, $3(6.1 \%)$ on fentanyl patch and $1(2 \%)$ on morphine and methadone. This means 33 $(66.3 \%)$ patients were on an opioid other than oxycodone. Of the 16 on oxycodone, 12 were male ( $52 \%$ of gender population) compared to 4 female ( $15.4 \%$ of gender population), $\mathrm{P}=0.013$. Five $(10.2 \%)$ patients had high SOAPP-R scores; 3 (13\%) of the males had a high SOAPP-R score compared to $2(7.7 \%)$ females. Regarding males on oxycodone, 3/12 (25\%) had high SOAPP-R scores; $1 / 4$ (25\%) females on oxycodone had a high SOAPP-R score. As age increased, the number of male patients using oxycodone trended upward. Older males on oxycodone had a higher SOAPP-R score compared to younger patients. No patient had prescriptions by more than one prescriber; 45 $(91.9 \%)$ patients had no pain contract. Urine drug screen was done in $11(22.5 \%)$ patients.

Conclusion: Prescription opioid drug abuse is not uncommon in primary care clinics. Use of the SOAPP-R, along with other appropriate patient evaluations, can help primary care providers identify high-risk behavior and decrease opioid abuse.

\section{An Interdisciplinary Process Change: Conversion of PICC Line Capping Solution From Heparin to Normal Saline}

Angela Colella, Brandon Bodager, Frank Spexarth, Natalie McDonough, Deb Kastenholz, Paul Grebe

Departments of Pharmacy, Radiology and Nursing, Aurora St. Luke's Medical Center, Aurora Health Care; AMG Imaging Services

Background: Heparin-induced thrombocytopenia (HIT) and HIT with thrombosis syndrome (HITTS) are serious conditions. Patients are at increased risk for developing HIT/HITTS with any exposure to heparin, even intravenous line flushes. Patients may be exposed to heparin multiple times each day when they have a peripherally inserted central catheter (PICC) that is flushed and capped with heparin. At Aurora Health Care, heparin is the standard capping solution for PICCs, but with a recent switch to positive pressurized caps, normal saline may be a capping option that reduces patient exposure to heparin.

Purpose: To reduce heparin exposure at a single hospital by replacing heparin with normal saline $(0.9 \%$ sodium chloride) as the standard PICC capping solution.

Methods: We implemented an interdisciplinary pilot process change and evaluated whether normal saline was noninferior to heparin for maintaining PICC patency. Primary outcome measurements of patency include alteplase use due to occlusion. Secondary objectives were any change in invasive line infection rates and cost comparisons. The baseline patency rate for the hospital was derived from data extracted through a retrospective chart review from October 2013 through October 2014.

Results: Our baseline patency rate was $65.42 \%(\mathrm{~N}=3,095)$; $33 \%$ of all PICC lines placed during the 12-month period were treated with alteplase. Patency rates during our pilot period were based on retrospective chart reviews of patients with PICC lines placed from February to May 2015. During this period, a total of 979 PICC lines were evaluated. Of these, $30.4 \%$ were treated with alteplase. This resulted in a pilot patency rate of $69.5 \%$, which is $4.1 \%$ greater than our baseline patency rate. Given the prespecified noninferiority margin of $5 \%$, our pilot demonstrates that normal saline is noninferior to heparin for maintaining PICC line patency when used with positive pressurized caps $(\alpha<0.05)$. Although number of infections increased $16 \%$, only $9 \%$ with line infections were part of our pilot. The estimated yearly cost of both capping solutions is equal, at approximately $\$ 3,960$ per our baseline 